outcome expenses. Serious reactions, such as purple glove syndrome, have not been reported with IV fosphenytoin.

Purple glove syndrome with IV phenytoin is reported in $5.9 \%$ of 152 adult patients treated in a 3-month period at the Mayo Clinic.(O'Brien TJ, Cascino GD, So EL, Hanna DR. Incidence and clinical consequence of the purple glove syndrome in patients receiving intravenous phenytoin. Neurology Oct 1998;51:1034-1039). (Reprints: Dr Gregory D Cascino, Department of Neurology, Mayo Clinic, 200 First Street SW, Rochester, MN 55905). Purple glove syndrome (PGS) is a progressive development of edema, discoloration, and pain after IV phenytoin. Elderly patients and those receiving large, multiple doses are particularly at risk. Most patients respond to conservative therapy.

\title{
LEARNING DISORDERS
}

\section{BRAIN LOCALIZATION OF MUSIC PROCESSING}

Music processing ability was studied in 65 right-handed patients following unilateral temporal cortectomy and compared to 24 matched normal controls at the Clinique Neurologique, Ponchaillou, Rennes, France; Departement de Psychologie, Universite de Montreal; and Centre de Reserche, Centre Hospitalier Cote-des-Neiges, Montreal, Quebec, Canada. Sequences of simple musical phrases were presented, with variations in either pitch or timing, to test ability to recognize changes in note intervals, rhythms, and metres. Deficits in discrimination of melodies were associated with right temporal lesions, and impairment of interval processing occurred with left temporal cortectomy. Pitch and timing were particularly affected by excision of the posterior superior temporal gyrus, part of the auditory cortex. Damage to the anterior part of the superior temporal gyrus caused impairment of metric processing, with preservation of rhythm. (Liegeois-Chauvel C, Peretz I, Babai M, Laguitton V, Chauvel P. Contribution of different cortical areas in the temporal lobes to music processing. Brain Oct 1998;121:1853-1867). (Respond: Catherine Liegeois-Chauvel, Laboratoire de Neurophysiologie et Neuropsychologie, INSERM CJF97-06, Universite de la Mediterranee, Faculte de Medicine, 27 Bd Jean Moulin-13385 Marseille Cedex, France).

COMMENT. The positive effects of music on learning in children are supported by neuroanatomical and electrophysiological studies, using high resolution PET scanners, EEG analysis, and observations of patients undergoing temporal cortectomy. Platel, Frackowiak and coworkers, in PET studies, found the left hemisphere dominant for rhythm, tune recognition, and pitch perception, while the right hemisphere is involved in timbre or quality of tone perception (Ped Neur Briefs April 1997;11:29-30). Listening to Mozart induces cortical right frontal and left hemisphere cerebral activity and enhances spatial-temporal reasoning (Ped Neur Briefs April 1998;4:32); it also lessens epileptic activity in the EEG of patients with epilepsy, both left or right temporal lobe focal discharges being suppressed (Ped Neur Briefs July 1998;7:52-53).

Meter is usually defined as the recurrent rhythmical pattern of notes, accents, and beats per measure in music. The above neuroanatomical study dissociates meter and rhythm, identifying separate cerebral locations for these cognitive musical processes. The metric organization of a sequence, involving the anterior part of the superior temporal gyrus, is not dependent on rhythmical organization or relative duration of notes. Functional MRI studies and music appreciation in children with ADHD would be of interest (Brain Dev Sept 1998;437) 